\title{
Beneficial Effects of Long-Term CPAP Treatment on Sleep Quality and Blood Pressure in Adherent Subjects With Obstructive Sleep Apnea
}

\author{
Mei-Chen Yang MD, Yi-Chih Huang MD, Chou-Chin Lan MD PhD, Yao-Kuang Wu MD, and \\ Kuo-Feng Huang MD PhD
}

\begin{abstract}
BACKGROUND: Obstructive sleep apnea (OSA) is associated with increased risk of cardiovascular diseases. Although CPAP is the first treatment choice for moderate-to-severe OSA, acceptance of and adherence to CPAP remain problematic. High CPAP adherence is generally defined as $\geq 4 \mathrm{~h}$ of use/night for $\geq 70 \%$ of the nights monitored. We investigated the long-term beneficial effects of CPAP on sleep quality and blood pressure in subjects with moderate-to-severe OSA according to high or low CPAP adherence. METHODS: We retrospectively analyzed 121 subjects with moderate-to-severe OSA from August 2008 to July 2012. These subjects were divided into 3 groups: (1) no CPAP treatment $(n=29)$, (2) low CPAP adherence $(n=28)$, and (3) high CPAP adherence $(n=$ 64). All subjects were followed up for at least $1 \mathrm{y}$. The 3 groups were compared regarding anthropometric and polysomnographic variables, presence of cardiovascular comorbidities, and blood pressure at baseline and at the last follow-up. RESULTS: The no-treatment group showed significant increases in oxygen desaturation index and blood pressure. The high-adherence group showed significant improvement in daytime sleepiness, apnea-hypopnea index (AHI), oxygen desaturation index, and blood pressure. Although the AHI was also significantly decreased after CPAP treatment in the lowadherence group, blood pressure remained unchanged. CONCLUSIONS: CPAP treatment had beneficial effects on both sleep quality and blood pressure only in subjects with OSA and high CPAP adherence who used CPAP for $\geq 4 \mathrm{~h} /$ night for $\geq 70 \%$ of nights monitored. Subjects with low CPAP adherence received beneficial effects on AHI, but not blood pressure. Key words: obstructive sleep apnea; CPAP; sleep quality, blood pressure. [Respir Care 2015;60(12):1810-1818. (C) 2015 Daedalus Enterprises]
\end{abstract}

\section{Introduction}

The incidence of obstructive sleep apnea (OSA) increases with age. ${ }^{1}$ It is known that OSA is a considerable risk factor

\footnotetext{
Drs Yang, Huang, Lan, and Wu are affiliated with the Division of Pulmonary Medicine, Department of Internal Medicine, and Dr Huang is affiliated with the Division of Neurosurgery, Department of Surgery, Taipei Tzu Chi Hospital, Buddhist Tzu Chi Medical Foundation, New Taipei City, Taiwan. Drs Yang, Lan, Wu, and Huang are also affiliated with the School of Medicine, Tzu Chi University, Hualien, Taiwan.

The authors have disclosed no conflicts of interest.

Correspondence: Kuo-Feng Huang MD PhD, School of Medicine, Tzu Chi University, Hualien 97004, Taiwan; Division of Neurosurgery, Department of Surgery, Taipei Tzu Chi Hospital, Buddhist Tzu Chi Medical Foundation, 289 Jianguo Road, Xindian District, New Taipei City 23143, Taiwan. E-mail: kuofeng1234@gmail.com.
}

DOI: $10.4187 /$ respcare.04199 for both cardiovascular and cerebrovascular diseases. ${ }^{2}$ CPAP treatment is the standard for managing OSA and has been proven to reduce the apnea-hypopnea index (AHI), improve sleep quality in subjects with OSA, ${ }^{3}$ and decrease the risk for cardiovascular comorbidities. ${ }^{4}$ An increase in blood pressure has a high correlation with OSA, and CPAP lowers the mortality rate in subjects with severe OSA. ${ }^{5}$ However, the limitations of CPAP treatment lie mainly in acceptance and adherence. CPAP adherence is reported in up to $50 \%$ of subjects with OSA. ${ }^{4}$ Failure to adhere to CPAP therapy may occur in up to $25-50 \%$ of patients, with patients typically abandoning therapy within the first 4 weeks of treatment. ${ }^{6}$

The differences in CPAP adherence are noted in many studies with various outcomes, but the long-term effects of CPAP have not been studied according to different CPAP adherence in detail. Thus, the purpose of this study was to compare the long-term effects of CPAP on sleep quality and blood pressure among CPAP-resistant, CPAP-adherent, and CPAP-nonadherent subjects. 


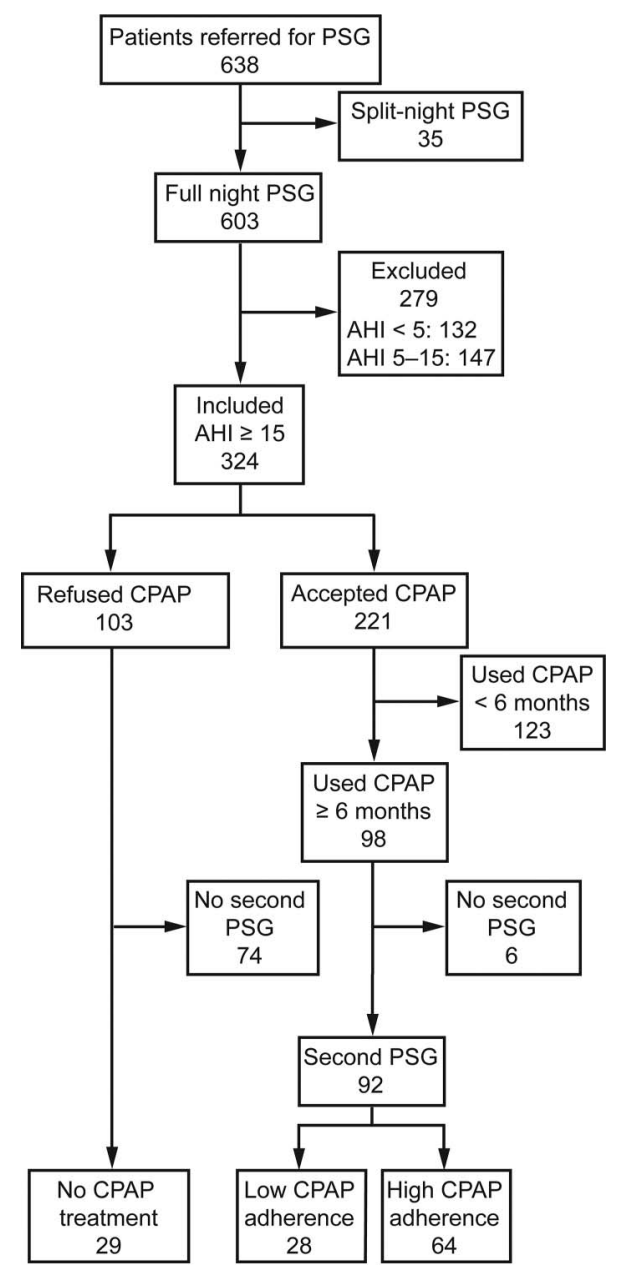

Fig. 1. Flow chart. PSG = polysomnography; $\mathrm{AHI}=$ apnea-hypopnea index.

\section{Methods}

\section{Study Subjects}

This study was approved by the institutional review board at Taipei Tzu Chi Hospital of the Buddhist Tzu Chi Medical Foundation (registration code 00-IRB-011-X). We retrospectively reviewed the medical charts of subjects with moderate-to-severe OSA between August 2008 and July 2012. All subjects' information was anonymized and de-identified before analysis.

Figure 1 demonstrates the flow chart for subject selection. Initially, there were 638 patients referred for overnight polysomnography (PSG) to rule out OSA. None of the patients were diagnosed as having OSA previously. Thirty-five patients who underwent split-night PSG were excluded. Therefore, 603 patients entered the full-night PSG study. Subsequently, 132 patients with PSG results showing non-apnea $(\mathrm{AHI}<5$ episodes/h) and 147 patients

\section{QUICK LOOK}

\section{Current knowledge}

Obstructive sleep apnea (OSA) is associated with an increased risk of cardiovascular disease. Although CPAP is the first treatment choice for moderate-to-severe OSA, acceptance of and adherence to CPAP therapy is poor. High CPAP adherence is generally defined as $\geq 4 \mathrm{~h}$ of use/night for $\geq 70 \%$ of monitored nights.

\section{What this paper contributes to our knowledge}

CPAP treatment demonstrated beneficial effects both on sleep quality and blood pressure in subjects with OSA and high CPAP adherence. Subjects with low CPAP adherence received beneficial effects on the apnea-hypopnea index, but not blood pressure. Techniques to improve CPAP adherence are needed to maximize treatment benefits.

with an AHI of 5-15 episodes/h were also excluded. Thus, there were 324 patients with an AHI of $\geq 15$ episodes/h.

Among these 324 patients with moderate-to-severe OSA, there were 103 patients who refused CPAP treatment and 221 patients who accepted CPAP treatment. One-hundred twenty-three patients who accepted CPAP were excluded because they used CPAP for $<6$ months. Thus, there were 98 subjects who accepted and used CPAP for $>6$ months. Patients who had no second PSG examination at follow-up were also excluded including 6 of $98 \mathrm{CPAP}$ acceptors and 74 of 103 CPAP resisters. Finally, 121 subjects with moderate-to-severe OSA were included in the final analysis. Twenty-nine subjects who refused CPAP composed the no-treatment group, and 28 and 64 subjects who accepted CPAP composed the low- and high-adherence group, respectively.

\section{Anthropometric Measurements and Demographic Data}

We collected the baseline clinical characteristics, including age, sex, neck circumference, body mass index (BMI, $\mathrm{kg} / \mathrm{m}^{2}$ ), smoking status, alcohol consumption, and cardiovascular comorbidities (including hypertension, coronary artery disease, and cerebrovascular accident). Morning and evening systolic (SBP) and diastolic (DBP) blood pressure were also checked and recorded before and after each PSG examination.

\section{Blood Pressure Measurement}

Blood pressure was measured while subjects were supine by technicians using an automated sphygmomanom- 
eter (Vital Signs Monitor 300 Series, Welch Allyn Skaneateles Falls, New York) with an optimal cuff. The automated sphygmomanometer was regularly calibrated every year. Evening blood pressure was measured after 15 min of rest before sleep onset, and morning blood pressure was measured immediately upon awakening with the subjects still attached to all PSG equipment. Two consecutive blood pressure readings were made on each occasion, separated by $5 \mathrm{~min}$, and the results were averaged as both the evening and morning blood pressure readings. Mean arterial blood pressure was calculated using usual method: mean arterial blood pressure $=1 / 3 \mathrm{SBP}+2 / 3 \mathrm{DBP}$.

\section{Assessment of Excessive Daytime Sleepiness}

Before the overnight PSG study, excessive daytime sleepiness was evaluated at the sleep center using the Epworth Sleepiness Scale (ESS). ${ }^{7,8}$ It was defined as an ESS score of $>11$.

\section{Sleep Parameters}

A standard overnight PSG was performed by trained sleep technicians who had received appropriate training from the Taiwan Society of Sleep Medicine and had at least $1 \mathrm{y}$ of experience. The PSG was recorded for at least $6 \mathrm{~h}$ with standard monitoring, including electroencephalography, electrooculography, chin and bilateral anterior tibialis surface electromyography, electrocardiography, air flow through the nose and mouth by thermistor, thoracoabdominal movements by respiratory inductive plethysmography, position sensor on the respiratory inductive plethysmography, snore sensor, and $\mathrm{S}_{\mathrm{pO}_{2}}$ simultaneously. PSG data were analyzed by manual scoring of every 30-s epoch. Sleep stage was scored by trained sleep technicians according to the standard criteria of Rechtschaffen and Kales. ${ }^{9}$ Apnea events were categorized into obstructive apnea, central apnea, mixed apnea, or hypopnea events. An apnea event was defined as the $80-100 \%$ reduction of air flow for at least $10 \mathrm{~s}$. Obstructive apnea was defined as the requisite reduction of $80-100 \%$ of air flow for at least $10 \mathrm{~s}$ with continued respiratory effort recorded in the chest and abdomen movement channels. Central apnea was defined as a cessation of both air flow and respiratory effort for at least $10 \mathrm{~s}$. Mixed apnea was defined by a period of both air flow and respiratory effort cessation, followed by a period of continued air-flow cessation despite gradually increasing respiratory effort. A hypopnea event was defined as at least $50 \%$ reduction of air flow for at least $10 \mathrm{~s}$ or at least $30 \%$ reduction of air flow for at least $10 \mathrm{~s}$ compared with baseline and associated with at least $3 \%$ oxygen desaturation or with an electroencephalogram arousal. The AHI was calculated as the total number of apnea and hypopnea events/h. The oxygen desaturation index (ODI) was calculated as the number of $\geq 3 \%$ desaturations/h. Sleep efficiency was defined as the fraction of total sleep time to total recording time.

\section{CPAP Acceptance and Adherence}

All subjects were offered CPAP treatment. CPAP acceptance refers to the subjects who meet the selection criteria for CPAP treatment and were willing to try CPAP for use at home. ${ }^{10,11} \mathrm{CPAP}$ adherence refers to the subjects who used CPAP and delivered a pre-set level over a given time period. ${ }^{10,11}$ Subjects were routinely followed up every 3 months at the out-patient clinic. According to the policy of our sleep center, we routinely downloaded objective CPAP use data (recorded by the device software) at each visit. The CPAP use data included percentage of days used, percentage of nights during which CPAP was used for $\geq 4 \mathrm{~h}$, and the overall mean hours of use/night. High CPAP adherence was defined as $\geq 4 \mathrm{~h}$ of CPAP use/night for $\geq 70 \%$ of the nights monitored. ${ }^{12-14}$ Subjects who did not meet these levels of CPAP use were defined as having low CPAP adherence.

\section{Statistical Analysis}

SAS 9.2 (SAS Institute, Cary, North Carolina) was used for the statistical analysis. The continuous variables (ie, demographics, ESS scores, overnight PSG data, blood pressure data, and CPAP adherence hours) were presented as mean \pm $\mathrm{SD}$. The categorical variables (ie, sex, disease history, excessive daytime sleepiness, and smoking and alcohol habits) were expressed by count and percentage. For comparisons of the 3 treatment groups, one-way analysis of variance was performed to examine the continuous variables at baseline. A chi-square test was used to examine the categorical variables. When a significant difference between groups was apparent, multiple comparisons were performed using the Bonferroni procedure with type- 1 error adjustment. To determine the difference between baseline and follow-up within the treatment groups, a paired $t$ test was performed for continuous variables, and the McNemar test was performed for categorical variables. All statistical assessments were evaluated at a 2-sided $\alpha$ level of .05 .

\section{Results}

\section{Demographics of Study Population}

The study analyzed 121 subjects with moderate-to-severe OSA; there were 103 males $(85.1 \%)$ and 18 females $(14.9 \%)$ (Table 1). There was no difference in neck circumference, BMI, smoking status, alcohol consumption, cardiovascular disease (including hypertension, cerebrovascular accident, and coronary artery disease), excessive 
Table 1. Baseline Characteristics of the 3 Groups

\begin{tabular}{|c|c|c|c|c|}
\hline Characteristic & $\begin{array}{l}\text { No Treatment } \\
\quad(n=29)\end{array}$ & $\begin{array}{l}\text { Low Adherence } \\
\quad(n=28)\end{array}$ & $\begin{array}{l}\text { High Adherence } \\
\quad(n=64)\end{array}$ & $P$ \\
\hline Age, y & $55.9 \pm 13.4$ & $49.7 \pm 12.5$ & $57.2 \pm 12.7 *$ & $.037 \dagger$ \\
\hline Sex & & & & .07 \\
\hline Female & $8(27.6)$ & $2(7.1)$ & $8(12.5)$ & \\
\hline Male & $21(72.4)$ & $26(92.9)$ & $56(87.5)$ & \\
\hline Neck circumference, $\mathrm{cm}$ & $40.7 \pm 4.1$ & $40.2 \pm 3.5$ & $40.5 \pm 3.2$ & .87 \\
\hline BMI, $\mathrm{kg} / \mathrm{m}^{2}$ & $30.3 \pm 4.4$ & $28.5 \pm 5.2$ & $29.1 \pm 4.6$ & .33 \\
\hline Smoking staus & $7(24.1)$ & $8(28.6)$ & $22(34.4)$ & .59 \\
\hline Alcohol consumption & $9(31)$ & $9(32.1)$ & $24(37.5)$ & .79 \\
\hline Hypertension & $17(58.6)$ & $12(42.9)$ & $34(53.1)$ & .48 \\
\hline Cardiovascular disease & $6(20.7)$ & $5(17.9)$ & $17(26.6)$ & .62 \\
\hline Cerebrovascular accident & $2(6.9)$ & $1(3.6)$ & $3(4.7)$ & .84 \\
\hline \multicolumn{5}{|l|}{ Daytime sleepiness } \\
\hline ESS score & $11.0 \pm 4.1$ & $11.6 \pm 4.4$ & $11.1 \pm 5.6$ & .85 \\
\hline Excessive daytime sleepiness & $17(58.6)$ & $18(64.3)$ & $37(57.8)$ & .84 \\
\hline \multicolumn{5}{|l|}{ Overnight polysomnogram } \\
\hline Sleep efficiency, \% & $79.3 \pm 13.7$ & $80.8 \pm 15.3$ & $79.7 \pm 12$ & .91 \\
\hline Stage $1, \%$ & $30.6 \pm 15.7$ & $30.9 \pm 16.8$ & $37.7 \pm 18.9$ & .10 \\
\hline Stage $2, \%$ & $56.3 \pm 14.7$ & $56.0 \pm 18.0$ & $50.9 \pm 17.3$ & .23 \\
\hline Stage $34, \%$ & $4.2 \pm 12.1$ & $1.2 \pm 3.1$ & $1.3 \pm 3.3$ & .13 \\
\hline REM, \% & $9.0 \pm 6.0$ & $11.8 \pm 7.9$ & $10.1 \pm 6.1$ & .25 \\
\hline AHI, episodes/h & $47.8 \pm 19.6$ & $52.0 \pm 23.3$ & $54.8 \pm 21.2$ & .35 \\
\hline ODI, episodes/h & $35.0 \pm 21.4$ & $39.3 \pm 26.9$ & $46.8 \pm 24.8$ & .09 \\
\hline \multicolumn{5}{|l|}{ Evening blood pressure, $\mathrm{mm} \mathrm{Hg}$} \\
\hline SBP & $123.8 \pm 13.1$ & $125.9 \pm 14$ & $126.5 \pm 13.4$ & .68 \\
\hline DBP & $78.5 \pm 10.2$ & $80.5 \pm 9.4$ & $79.3 \pm 11$ & .77 \\
\hline Mean arterial blood pressure & $93.6 \pm 10.4$ & $95.6 \pm 10.4$ & $95 \pm 11.2$ & .77 \\
\hline \multicolumn{5}{|l|}{ Morning blood pressure, $\mathrm{mm} \mathrm{Hg}$} \\
\hline SBP & $125.1 \pm 12.8$ & $128.3 \pm 11.5$ & $133.3 \pm 14.9 \ddagger$ & $.02 \dagger$ \\
\hline DBP & $80.2 \pm 10.1$ & $85.6 \pm 9.1$ & $84.6 \pm 14.6$ & .21 \\
\hline Mean arterial blood pressure & $95.2 \pm 10.4$ & $99.8 \pm 9.1$ & $100.8 \pm 13.6$ & .11 \\
\hline $\begin{array}{l}\text { Values are expressed as mean } \pm \text { SD or } n \text { ( } \\
* \text { Significantly different compared with the } \\
\dagger P<.05 \text { indicates a significant difference } \\
¥ \text { Significantly different compared with the } \\
\text { BMI }=\text { body mass index } \\
\text { ESS }=\text { Epworth Sleepiness Scale } \\
\text { PSG }=\text { polysomnography } \\
\text { REM }=\text { rapid eye movement } \\
\text { AHI }=\text { apnea-hypopnea index } \\
\text { ODI }=\text { oxygen desaturation index } \\
\text { SBP }=\text { systolic blood pressure } \\
\text { DBP }=\text { diastolic blood pressure }\end{array}$ & $\begin{array}{l}\text { p. } \\
\text { nent groups. }\end{array}$ & & & \\
\hline
\end{tabular}

daytime sleepiness, and PSG variables. The low-adherence group was significantly younger than the high-adherence group $(49.7 \pm 12.5$ vs $57.2 \pm 12.7 \mathrm{y}, P=.037)$. The no-treatment group showed a significant lower morning SBP than the high-adherence group $(125.1 \pm 12.8 \mathrm{vs}$ $133.3 \pm 14.9 \mathrm{~mm} \mathrm{Hg}, P=.02$ ).

\section{Sleep Quality and Blood Pressure at Baseline and Follow-Up}

Table 2 summarizes the changes in BMI, daytime sleepiness, PSG variables, and blood pressure in the 3 groups at baseline and follow-up. The BMI was not significantly different in the 3 groups at follow-up. The ESS (11.1 \pm 5.6 vs $9.5 \pm 4.9, P=.006)$ and excessive daytime sleepiness $(57.8 \%$ vs $37.5 \%, P=.004)$ of the high-adherence group decreased significantly. The sleep efficiency of the high-adherence group significantly decreased by $3.6 \%$ from baseline $(79.7 \pm 12 \%$ vs $76.1 \pm 15.9 \%, P=.046)$. Stage 1 sleep in the high-adherence group significantly decreased by $5.9 \%$ from baseline $(37.7 \pm 18.9 \%$ vs $31.8 \pm 14.6 \%$, $P=.01)$. The rapid eye movement sleep of the no-treatment group significantly increased by $3.2 \%$ from baseline 
EfFects of CPAP on Sleep Quality and Blood Pressure in OSA

Table 2. Sleep Quality and Blood Pressures at Baseline and Follow-Up in the 3 Groups

\begin{tabular}{|c|c|c|c|c|c|c|c|c|c|}
\hline \multirow[t]{2}{*}{ Variable } & \multicolumn{3}{|c|}{$\begin{array}{l}\text { No Treatment } \\
\quad(n=29)\end{array}$} & \multicolumn{3}{|c|}{$\begin{array}{l}\text { Low Adherence } \\
\quad(n=28)\end{array}$} & \multicolumn{3}{|c|}{$\begin{array}{l}\text { High Adherence } \\
\quad(n=64)\end{array}$} \\
\hline & Baseline & Follow-Up & $P$ & Baseline & Follow-Up & $P$ & Baseline & Follow-Up & $P$ \\
\hline BMI, $\mathrm{kg} / \mathrm{m}^{2}$ & $30.3 \pm 4.4$ & $29.9 \pm 4.6$ & .26 & $28.5 \pm 5.2$ & $28.4 \pm 5.2$ & .79 & $29.1 \pm 4.6$ & $29.1 \pm 4.8$ & .86 \\
\hline \multicolumn{10}{|l|}{ Daytime sleepiness } \\
\hline ESSscore & $11.0 \pm 4.1$ & $11.7 \pm 5.3$ & .34 & $11.6 \pm 4.4$ & $11.4 \pm 5$ & .76 & $11.1 \pm 5.6$ & $9.5 \pm 4.9$ & $.006^{*}$ \\
\hline Excessive daytime sleepiness & $17(58.6)$ & $19(65.5)$ & .73 & $18(64.3)$ & $19(67.9)$ & $>.99$ & $37(57.8)$ & $24(37.5)$ & $.004 *$ \\
\hline \multicolumn{10}{|l|}{ Overnight PSG } \\
\hline Sleep efficiency, $\%$ & $79.3 \pm 13.7$ & $77.2 \pm 14.3$ & .48 & $80.8 \pm 15.3$ & $78.8 \pm 16.5$ & .54 & $79.7 \pm 12$ & $76.1 \pm 15.9$ & $.046^{*}$ \\
\hline Stage $1, \%$ & $30.6 \pm 15.7$ & $30.1 \pm 14.3$ & .84 & $30.9 \pm 16.8$ & $30.1 \pm 17.3$ & .71 & $37.7 \pm 18.9$ & $31.8 \pm 14.6$ & $.01 *$ \\
\hline Stage $2, \%$ & $56.3 \pm 14.7$ & $55.9 \pm 12.8$ & .88 & $56.0 \pm 18.0$ & $56.8 \pm 16.5$ & .79 & $50.9 \pm 17.3$ & $55.7 \pm 12.9$ & .051 \\
\hline Stage $34, \%$ & $4.2 \pm 12.1$ & $1.8 \pm 4.5$ & .35 & $1.2 \pm 3.1$ & $1.7 \pm 3.2$ & .58 & $1.3 \pm 3.3$ & $1.8 \pm 3.6$ & .40 \\
\hline REM, \% & $9.0 \pm 6.0$ & $12.2 \pm 6.7$ & $.046^{*}$ & $11.8 \pm 7.9$ & $11.4 \pm 6.9$ & .83 & $10.1 \pm 6.1$ & $10.7 \pm 6.8$ & .48 \\
\hline AHI, episodes/h & $47.8 \pm 19.6$ & $50.2 \pm 21.5$ & .45 & $52.0 \pm 23.3$ & $42.0 \pm 27.0$ & $.03^{*}$ & $54.8 \pm 21.2$ & $39.7 \pm 21.4$ & $<.001 *$ \\
\hline ODI, episodes $/ \mathrm{h}$ & $35.0 \pm 21.4$ & $42.7 \pm 24.1$ & $.02 *$ & $39.3 \pm 26.9$ & $35.8 \pm 26.8$ & .33 & $46.8 \pm 24.8$ & $33.9 \pm 20.5$ & $<.001^{*}$ \\
\hline \multicolumn{10}{|l|}{ Evening blood pressure, $\mathrm{mm} \mathrm{Hg}$} \\
\hline SBP & $123.8 \pm 13.1$ & $130.1 \pm 13.9$ & $.01 *$ & $125.9 \pm 14$ & $130.8 \pm 12.3$ & .11 & $126.5 \pm 13.4$ & $128.1 \pm 13.4$ & .34 \\
\hline DBP & $78.5 \pm 10.2$ & $79.0 \pm 9.7$ & .78 & $80.5 \pm 9.4$ & $82.5 \pm 9.8$ & .31 & $79.3 \pm 11$ & $76.3 \pm 10.4$ & $.01 *$ \\
\hline Mean arterial blood pressure & $93.6 \pm 10.4$ & $96.1 \pm 10.7$ & .20 & $95.6 \pm 10.4$ & $98.6 \pm 9.7$ & .16 & $95 \pm 11.2$ & $93.6 \pm 10.5$ & .26 \\
\hline \multicolumn{10}{|l|}{ Morning blood pressure, $\mathrm{mm} \mathrm{Hg}$} \\
\hline SBP & $125.1 \pm 12.8$ & $134.6 \pm 12.1$ & $<.001 *$ & $128.3 \pm 11.5$ & $134.1 \pm 14.8$ & .067 & $133.3 \pm 14.9$ & $127.2 \pm 12.5$ & $.001 *$ \\
\hline DBP & $80.2 \pm 10.1$ & $84.4 \pm 8.1$ & $.006^{*}$ & $85.6 \pm 9.1$ & $86.5 \pm 10.7$ & .73 & $84.6 \pm 14.6$ & $81.1 \pm 11.3$ & $.02 *$ \\
\hline Mean arterial blood pressure & $95.2 \pm 10.4$ & $101.2 \pm 8.9$ & $<.001^{*}$ & $99.8 \pm 9.1$ & $102.3 \pm 11.6$ & .33 & $100.8 \pm 13.6$ & $96.6 \pm 10.8$ & $.004 *$ \\
\hline $\begin{array}{l}\text { Values are expressed as mean } \pm \mathrm{SD} \text { or } n(c) \\
* P<.05 \text { indicates a significant difference } \\
\text { BMI }=\text { body mass index } \\
\text { ESS }=\text { Epworth Sleepiness Scale } \\
\text { PSG }=\text { polysomnography } \\
\text { REM }=\text { rapid eye movement } \\
\text { AHI }=\text { apnea-hypopnea index } \\
\text { ODI }=\text { oxygen desaturation index } \\
\text { SBP }=\text { systolic blood pressure } \\
\text { DBP }=\text { diastolic blood pressure }\end{array}$ & efore and after treat & ment within treatme & nt groups. & & & & & & \\
\hline
\end{tabular}

$(9.0 \pm 6.0 \%$ vs $12.2 \pm 6.7 \%, P=.046)$. The AHI significantly decreased in both the low-adherence group $(52.0 \pm 23.3$ vs $42.0 \pm 27.0$ episodes $/ \mathrm{h}, P=.03)$ and high-adherence group $(54.8 \pm 21.2$ vs $39.7 \pm 21.4$ episodes/h, $P<.001)$. The ODI significantly decreased in the high-adherence group $(46.8 \pm 24.8$ vs $33.9 \pm 20.5$ episodes $/ \mathrm{h}, P<.001$ ), but increased in the no-treatment group $(35.0 \pm 21.4$ vs $42.7 \pm 24.1$ episodes $/ \mathrm{h}, P=.02)$.

The evening SBP $(123.8 \pm 13.1$ vs $130.1 \pm 13.9 \mathrm{~mm}$ $\mathrm{Hg}, P=.01)$ and the morning SBP $(125.1 \pm 12.8$ vs $134.6 \pm 12.1 \mathrm{~mm} \mathrm{Hg}, P<.001)$, DBP $(80.2 \pm 10.1 \mathrm{vs}$ $84.4 \pm 8.1 \mathrm{~mm} \mathrm{Hg}, P=.006)$, and mean arterial blood pressure $(95.2 \pm 10.4$ vs $101.2 \pm 8.9 \mathrm{~mm} \mathrm{Hg}, P<.001)$ significantly increased at follow-up in the no-treatment group . The evening DBP $(79.3 \pm 11.0$ vs $76.3 \pm 10.4 \mathrm{~mm}$ $\mathrm{Hg}, P=.01)$ and the morning $\mathrm{SBP}(133.3 \pm 14.9$ vs $127.2 \pm 12.5 \mathrm{~mm} \mathrm{Hg}, P=.001)$, DBP $(84.6 \pm 14.6$ vs $81.1 \pm 11.3 \mathrm{~mm} \mathrm{Hg}, P=.02)$, and mean arterial blood pressure $(100.8 \pm 13.6$ vs $96.6 \pm 10.8 \mathrm{~mm} \mathrm{Hg}, P=.004)$ significantly decreased at follow-up in the high-adherence group. Although there was no statistical significance, we still observed a trend of increasing blood pressure in the low-adherence group at follow-up (Table 2 and Fig. 2).

\section{CPAP Adherence}

Subjects in the high-adherence group had higher CPAP adherence than those in the low-adherence group (91.5 \pm $7.8 \%$ vs $53.4 \pm 16.5 \%, P<.001$ ) (Table 3 ). The mean hours of CPAP use were longer in the high-adherence group than in the low-adherence group $(6.5 \pm 1.1$ vs $3.9 \pm$ $1.1 \mathrm{~h}, P<.001)$. There were no significant differences in the total follow-up duration among the 3 groups. The CPAP adherence was significantly different between the highand low-adherence groups.

\section{Discussion}

In this study, we observed significant beneficial effects of long-term CPAP on sleep quality and blood pressure only in subjects with moderate-to-severe OSA and high CPAP adherence. If subjects with moderate-to-severe OSA did not receive CPAP treatment, their sleep quality and 

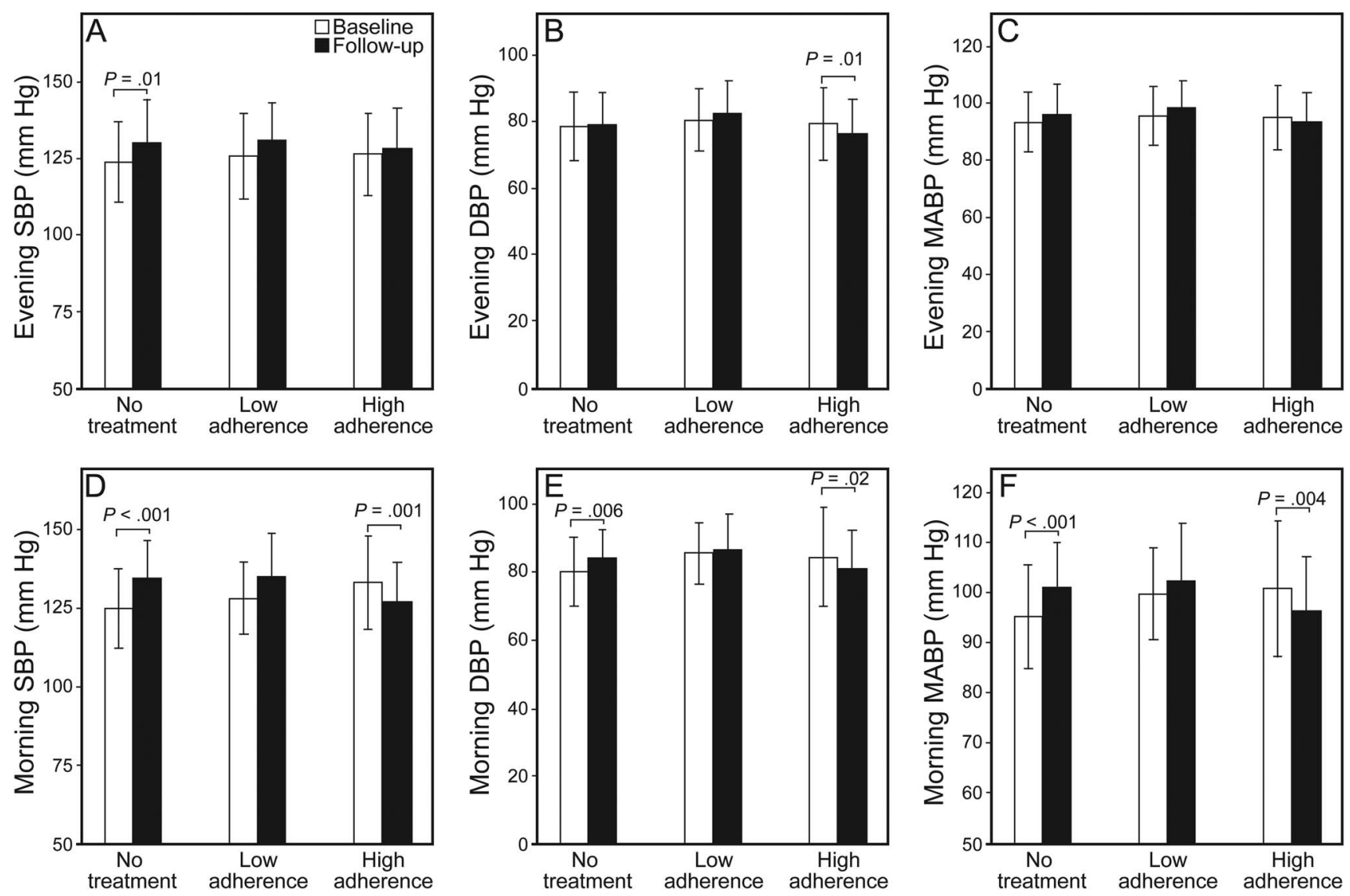

Fig. 2. Comparison of blood pressure at baseline and follow-up among the 3 groups. SBP $=$ systolic blood pressure; DBP $=$ diastolic blood pressure; MABP = mean arterial blood pressure.

Table 3. CPAP Adherence in the 3 Groups

\begin{tabular}{|c|c|c|c|c|}
\hline Variable & No Treatment $(n=29)$ & Low Adherence $(n=28)$ & High Adherence $(n=64)$ & $P$ \\
\hline Follow-up duration, mo & $14.9 \pm 3.8$ & $18.3 \pm 8.5$ & $18.8 \pm 7.6$ & .053 \\
\hline CPAP use, mo & NA & $14.6 \pm 7.2$ & $15.9 \pm 7.7$ & .44 \\
\hline CPAP adherence, $\%$ & NA & $53.4 \pm 16.5$ & $91.5 \pm 7.8$ & $<.001^{*}$ \\
\hline CPAP adherence, $\mathrm{h}$ & NA & $3.9 \pm 1.1$ & $6.5 \pm 1.1$ & $<.001^{*}$ \\
\hline \multicolumn{5}{|c|}{$\begin{array}{l}\text { Values are expressed as mean } \pm \mathrm{SD} \text {. } \\
* P<.05 \text { indicates a significant difference among the } 3 \text { treatment groups. } \\
\mathrm{NA}=\text { not applicable }\end{array}$} \\
\hline
\end{tabular}

blood pressure became worse over time. Although subjects with moderate-to-severe OSA and poor CPAP adherence had some beneficial effects on AHI, their blood pressure still had the potential to increase with time, leading to increasing risk of cardiovascular comorbidities. Better adherence to CPAP resulted in more beneficial effects in subjects with moderate-to-severe OSA.

\section{CPAP Acceptance in Subjects of Different Ages}

In this study, we noted that older subjects with OSA either completely refused CPAP treatment (no-treatment group, $55.9 \pm 13.4$ y old) or used CPAP very well (highadherence group, $57.2 \pm 12.7$ y old), in contrast to the younger subjects with OSA (low-adherence group, $49.7 \pm$ $12.5 \mathrm{y}$ old). This was also documented in our previous study. ${ }^{15}$ This interesting result showing that subjects in the low-adherence group were younger reveals that savvy and health concern seem more common in older subjects. We claim that this is also possibly related to socioeconomic status. In Taiwan, OSA patients must purchase CPAP equipment at their own expense because it is not covered by national or commercial insurance, and the cost of CPAP 
equipment is $2-3$ times higher than in other countries. ${ }^{16,17}$ Elderly patients with a higher economic status are able to pay for their health maintenance. However, some older patients with lower economic status choose not to treat OSA because they are struggling with costs of other agingrelated medical problems.

\section{BMI}

The BMI has been noted as being highly correlated with OSA severity and blood pressure. ${ }^{18-20}$ As indicated in Table 2, the BMI at baseline and follow-up showed no significant changes among the 3 groups, indicating that the changes in AHI, ODI, or blood pressure in our study were not related to the BMI changes.

\section{Daytime Sleepiness and CPAP}

CPAP treatment can improve excessive daytime sleepiness in patients with OSA both short- and long-term. Zhao et $\mathrm{al}^{21}$ reported significant improvement in excessive daytime sleepiness after 1 month of CPAP treatment in subjects with moderate-to-severe OSA and coronary heart diseases. In other previous studies, a dose-response effect on ESS scores was documented after 3 months of CPAP treatment. ${ }^{22,23}$ In another earlier study, Pietrzyk et al ${ }^{24}$ found a beneficial effect of $1 \mathrm{y}$ of CPAP treatment on excessive daytime sleepiness in subjects with OSA. Our findings were compatible with previous studies and also supported the dose-dependent response of excessive daytime sleepiness to long-term CPAP treatment. Subjects with poor CPAP adherence could not benefit from CPAP in relation to excessive daytime sleepiness.

\section{Sleep Quality and CPAP}

Cooke et $\mathrm{al}^{25}$ found that in mild-to-moderate Alzheimer's disease subjects with OSA, CPAP resulted in deeper sleep after just one night, with improvements maintained for 3 weeks. Loredo et $\mathrm{a}^{26}$ found that CPAP improved sleep quality in subjects with OSA by consolidating sleep, reducing stage 1 sleep, and improving rapid eye movement sleep, but had no significant effect on stage 2 sleep or slow-wave sleep. CPAP can improve the AHI and ODI. ${ }^{26,27}$ In our study, we also observed some improvements in sleep quality: reducing stage 1 sleep, AHI, and ODI in the high-adherence group and reducing AHI in the low-adherence group. However, the sleep efficiency of the highadherence group became worse because withdrawal from usual CPAP use made it difficult for them to maintain normal sleep. If patients with OSA do not receive adequate CPAP treatment, the OSA will become more severe.

\section{Blood Pressure and CPAP}

CPAP treatment is known to reduce the risk of fatal and nonfatal cardiovascular events, which are significantly increased by severe OSA. ${ }^{5}$ In a previous report, ${ }^{28}$ we found increasing evidence of greater cardiovascular risk for untreated mild OSA, and improving CPAP acceptance by subjects with mild OSA may be clinically important. However, the important issue is just how much CPAP use is enough to reduce blood pressure? Most previous studies (focused on the blood pressure-lowering effect of CPAP) used different definitions of CPAP adherence and thus make it difficult to draw conclusions. ${ }^{21,29-31}$ One earlier study, published in 2005, concluded that low CPAP use is better than no use in reducing OSA-related mortality. ${ }^{32}$ Another study in 2007 found a dose-dependent effect of CPAP on improvement of quality of life. ${ }^{33}$ Other studies also showed that a daily average of $3 \mathrm{~h}$ of CPAP use is sufficient to decrease DBP in subjects with severe OSA and hypertension. ${ }^{29,30}$ However, one report showed that CPAP therapy has little effect on reducing blood pressure in subjects with OSA. ${ }^{13}$ Until recently, there were 3 metaanalyses that focused on duration of CPAP use, and they found that both diurnal SBP and DBP were significantly reduced only with CPAP use for $\geq 4$ weeks and for $\geq 4$ h/night. ${ }^{13,14,34}$ In our present study, we used the same duration to define CPAP adherence ( $\geq 4 \mathrm{~h} /$ night for $\geq 70 \%$ of nights) and obtained comparable results showing the longterm beneficial effects of CPAP on blood pressure in subjects with moderate-to-severe OSA who adhere to CPAP treatment.

\section{Adherence Effect on Outcome}

The American Academy of Sleep Medicine states that adherence to CPAP treatment is crucial and that routine assessment is necessary. ${ }^{35-37}$ Although it is known that CPAP is an effective therapy for OSA and long-term adherence has a considerable influence on its effectiveness, only about half of patients with OSA have high long-term adherence. ${ }^{10}$ Our present study revealed that subjects with moderate-to-severe OSA and high CPAP adherence obtained beneficial effects in reducing cardiovascular comorbidities with lower blood pressure. How to improve CPAP acceptance and adherence is always problematic and should be addressed in the future.

\section{Possible Mechanism of CPAP on Blood Pressure}

CPAP improves endothelial function (not arterial stiffness) in minimally symptomatic OSA, which is a cardiovascular risk factor. ${ }^{38}$ Endothelial dysfunction is known to play a central role in the development of atherosclerosis and is associated with cardiovascular risk factors. ${ }^{39}$ Nich- 
oll et al ${ }^{40}$ reported that CPAP therapy had a correlation with down-regulation of renal renin-angiotensin system activity. A randomized controlled study also showed that CPAP significantly reduced plasma aldosterone concentration in subjects with OSA and hypertension. ${ }^{41}$ In addition, research has emphasized that thrombotic tendency and blood viscosity are possible mechanisms of disease in subjects with OSA and cardiovascular comorbidity. ${ }^{2}$

\section{Limitations of This Study}

First, our study had the inherent weakness of a retrospective design and a relatively small sample size. Larger, prospectively controlled studies are warranted to further study the effects of CPAP treatment on sleep quality, blood pressure, and long-term cardiovascular comorbidities in different patterns of CPAP use. Second, in our sleep center, we used a thermistor, not a nasal pressure transducer, to detect air flow, and we did not use an esophageal pressure sensor to detect respiratory effort-related arousal. This might result in an underestimation of the severity of OSA and consequently a misclassification of some subjects with moderate-to-severe OSA as mild. Third, we did not evaluate the impact of antihypertensive medicine, possible sleeping pill use, or other medical conditions that would affect blood pressure control (such as diabetes mellitus and hyperlipidemia). In addition, arbitrarily defining CPAP adherence using a 4-h cutoff value may not be optimal because subjects normally have varied sleep durations.

\section{Conclusions}

This study demonstrated that subjects with moderateto-severe OSA and high CPAP adherence obtained longterm beneficial effects in sleep quality and reduced cardiovascular comorbidities with lower blood pressure. Subjects with low CPAP adherence demonstrated beneficial effects on only AHI, but not blood pressure. Future studies are needed to identify the key obstacles and solutions to CPAP adherence. Strategies for improving CPAP adherence are still an issue.

\section{REFERENCES}

1. Prisant LM, Dillard TA, Blanchard AR. Obstructive sleep apnea syndrome. J Clin Hypertens 2006;8(10):746-750.

2. Toraldo DM, Peverini F, De Benedetto M, De Nuccio F. Obstructive sleep apnea syndrome: blood viscosity, blood coagulation abnormalities, and early atherosclerosis. Lung 2013;191(1):1-7.

3. Randerath WJ, Verbraecken J, Andreas S, Bettega G, Boudewyns A, Hamans E, et al. Non-CPAP therapies in obstructive sleep apnoea. Eur Respir J 2011;37(5):1000-1028.

4. Hussain SF, Irfan M, Waheed Z, Alam N, Mansoor S, Islam M. Compliance with continuous positive airway pressure (CPAP) therapy for obstructive sleep apnea among privately paying patients-a cross sectional study. BMC Pulm Med 2014;14:188.
5. Marin JM, Carrizo SJ, Vicente E, Agusti AG. Long-term cardiovascular outcomes in men with obstructive sleep apnoea-hypopnoea with or without treatment with continuous positive airway pressure: an observational study. Lancet 2005;365(9464):1046-1053.

6. Queiroz DL, Yui MS, Braga AA, Coelho ML, Küpper DS, Sander $\mathrm{HH}$, et al. Adherence of obstructive sleep apnea syndrome patients to continuous positive airway pressure in a public service. Braz J Otorhinolaryngol 2014;80(2):126-130.

7. Johns MW. A new method for measuring daytime sleepiness: the Epworth sleepiness scale. Sleep 1991;14(6):540-545.

8. Chen NH, Johns MW, Li HY, Chu CC, Liang SC, Shu YH, et al. Validation of a Chinese version of the Epworth sleepiness scale. Qual Life Res 2002;11(8):817-821.

9. Hori T, Sugita Y, Koga E, Shirakawa S, Inoue K, Uchida S, et al. Proposed supplements and amendments to 'A Manual of Standardized Terminology, Techniques and Scoring System for Sleep Stages of Human Subjects,' the Rechtschaffen \& Kales (1968) standard. Psychiatry Clin Neurosci 2001;55(3):305-310.

10. Anstead M, Phillips B, Buch K. Tolerance and intolerance to continuous positive airway pressure. Curr Opin Pulm Med 1998;4(6): 351-354.

11. Grunstein RR. Sleep-related breathing disorders. 5. Nasal continuous positive airway pressure treatment for obstructive sleep apnoea. Tho$\operatorname{rax}$ 1995;50(10):1106-1113.

12. Gay P, Weaver T, Loube D, Iber C, Positive Airway Pressure Task Force, Standards of Practice Committee, American Academy of Sleep Medicine. Evaluation of positive airway pressure treatment for sleep related breathing disorders in adults. Sleep 2006;29(3):381-401.

13. Fava C, Dorigoni S, Dalle Vedove F, Danese E, Montagnana M, Guidi GC, et al. Effect of CPAP on blood pressure in patients with OSA/hypopnea a systematic review and meta-analysis. Chest 2014; 145(4):762-771.

14. Montesi SB, Edwards BA, Malhotra A, Bakker JP. The effect of continuous positive airway pressure treatment on blood pressure: a systematic review and meta-analysis of randomized controlled trials. J Clin Sleep Med 2012;8(5):587-596.

15. Yang MC, Lin CY, Lan CC, Huang CY, Huang YC, Lim CS, et al. Factors affecting CPAP acceptance in elderly patients with obstructive sleep apnea in Taiwan. Respir Care 2013;58(9):1504-1513.

16. Simon-Tuval T, Reuveni H, Greenberg-Dotan S, Oksenberg A, Tal A, Tarasiuk A. Low socioeconomic status is a risk factor for CPAP acceptance among adult OSAS patients requiring treatment. Sleep 2009;32(4):545-552.

17. Tarasiuk A, Reznor G, Greenberg-Dotan S, Reuveni H. Financial incentive increases CPAP acceptance in patients from low socioeconomic background. PLoS ONE 2012;7(3):e33178.

18. Mehdad S, Hamrani A, El Kari K, El Hamdouchi A, El Mzibri M, Barkat A, et al. Prevalence of elevated blood pressure and its relationship with fat mass, body mass index and waist circumference among a group of Moroccan overweight adolescents. Obes Res Clin Pract 2013;7(4):e284-e289.

19. Morris LG, Kleinberger A, Lee KC, Liberatore LA, Burschtin O. Rapid risk stratification for obstructive sleep apnea, based on snoring severity and body mass index. Otolaryngol Head Neck Surg 2008; 139(5):615-618.

20. Zhang YX, Wang SR. Comparison of blood pressure levels among children and adolescents with different body mass index and waist circumference: study in a large sample in Shandong, China. Eur J Nutr 2014;53(2):627-634.

21. Zhao Q, Liu ZH, Luo Q, Zhao ZH, Zhang HL, Wang Y. Effects of continuous positive airway pressure on blood pressure and daytime sleepiness in obstructive sleep apnea patients with coronary heart diseases under optimal medications. Sleep Breath 2012;16(2):341347. 
22. Antic NA, Catcheside P, Buchan C, Hensley M, Naughton MT, Rowland S, et al. The effect of CPAP in normalizing daytime sleepiness, quality of life, and neurocognitive function in patients with moderate-to-severe OSA. Sleep 2011;34(1):111-119.

23. Weaver TE, Maislin G, Dinges DF, Bloxham T, George CF, Greenberg $\mathrm{H}$, et al. Relationship between hours of CPAP use and achieving normal levels of sleepiness and daily functioning. Sleep 2007;30(6): 711-719.

24. Pietrzyk A, Zgierska A, Pływaczewski R, Zieliński J. [The effect of long-term therapy of obstructive sleep apnea syndrome with CPAP for symptoms of daytime sleepiness]. Wiad Lek 1997;50(10-12): 295-299. Article in Polish.

25. Cooke JR, Ancoli-Israel S, Liu L, Loredo JS, Natarajan L, Palmer $\mathrm{BS}$, et al. Continuous positive airway pressure deepens sleep in patients with Alzheimer's disease and obstructive sleep apnea. Sleep Med 2009;10(10):1101-1106.

26. Loredo JS, Ancoli-Israel S, Kim EJ, Lim WJ, Dimsdale JE. Effect of continuous positive airway pressure versus supplemental oxygen on sleep quality in obstructive sleep apnea: a placebo-CPAP-controlled study. Sleep 2006;29(4):564-571.

27. Loredo JS, Ancoli-Israel S, Dimsdale JE. Effect of continuous positive airway pressure vs placebo continuous positive airway pressure on sleep quality in obstructive sleep apnea. Chest 1999;116(6):1545-1549.

28. Huang YC, Lin CY, Lan CC, Wu YK, Lim CS, Huang CY, et al. Comparison of cardiovascular co-morbidities and CPAP use in patients with positional and non-positional mild obstructive sleep apnea. BMC Pulm Med 2014;14:153.

29. Aihara K, Chin K, Oga T, Takahashi K, Hitomi T, Takegami M, et al. Long-term nasal continuous positive airway pressure treatment lowers blood pressure in patients with obstructive sleep apnea regardless of age. Hypertens Res 2010;33(10):1025-1031.

30. Chin K, Nakamura T, Takahashi K, Sumi K, Matsumoto H, Niimi A, et al. Falls in blood pressure in patients with obstructive sleep apnoea after long-term nasal continuous positive airway pressure treatment. J Hypertens 2006;24(10):2091-2099.

31. Varounis C, Katsi V, Kallikazaros IE, Tousoulis D, Stefanadis C, Parissis J, et al. Effect of CPAP on blood pressure in patients with obstructive sleep apnea and resistant hypertension: a systematic review and meta-analysis. Int J Cardiol 2014;175(1):195-198.
32. Campos-Rodriguez F, Peña-Griñan N, Reyes-Nuñez N, De la CruzMoron I, Perez-Ronchel J, De la Vega-Gallardo F, Fernandez-Palacin A. Mortality in obstructive sleep apnea-hypopnea patients treated with positive airway pressure. Chest 2005;128(2):624-633.

33. Mystakidou K, Parpa E, Tsilika E, Pathiaki M, Gennatas K, Smyrniotis V, Vassiliou I. The relationship of subjective sleep quality, pain, and quality of life in advanced cancer patients. Sleep 2007; 30(6):737-742.

34. Bratton DJ, Stradling JR, Barbé F, Kohler M. Effect of CPAP on blood pressure in patients with minimally symptomatic obstructive sleep apnoea: a meta-analysis using individual patient data from four randomised controlled trials. Thorax 2014;69(12):1128-1135.

35. Weaver TE, Grunstein RR. Adherence to continuous positive airway pressure therapy: the challenge to effective treatment. Proc Am Thorac Soc 2008;5(2):173-178.

36. Weaver TE, Sawyer AM. Adherence to continuous positive airway pressure treatment for obstructive sleep apnoea: implications for future interventions. Indian J Med Res 2010;131:245-258.

37. Weaver TE. Don't start celebrating-CPAP adherence remains a problem. J Clin Sleep Med 2013;9(6):551-552.

38. Kohler M, Craig S, Pepperell JC, Nicoll D, Bratton DJ, Nunn AJ, et al. CPAP improves endothelial function in patients with minimally symptomatic OSA: results from a subset study of the MOSAIC trial. Chest 2013;144(3):896-902.

39. Carvalho LS, Virginio VW, Panzoldo NB, Figueiredo VN, Santos SN, Modolo RG, et al. Elevated CETP activity during acute phase of myocardial infarction is independently associated with endothelial dysfunction and adverse clinical outcome. Atherosclerosis 2014; 237(2):777-783.

40. Nicholl DD, Hanly PJ, Poulin MJ, Handley GB, Hemmelgarn BR, Sola DY, Ahmed SB. Evaluation of continuous positive airway pressure therapy on renin-angiotensin system activity in obstructive sleep apnea. Am J Respir Crit Care Med 2014;190(5):572-580.

41. Lloberes P, Sampol G, Espinel E, Segarra A, Ramon MA, Romero $\mathrm{O}$, et al. A randomized controlled study of CPAP effect on plasma aldosterone concentration in patients with resistant hypertension and obstructive sleep apnea. J Hypertens 2014;32(8):1650-1657; discussion 1657. 